\title{
Revised instability domains of SPB and $\beta$ Cephei stars
}

\author{
A. Miglio ${ }^{1}$, J. Montalbán ${ }^{1}$ and M.-A. Dupret ${ }^{2}$ \\ ${ }^{1}$ Institut d'Astrophysique, Allée du 6 Août, 17, B-4000 Liège, Belgium \\ ${ }^{2}$ Observatoire de Paris, LESIA, CNRS UMR 8109, 92195 Meudon, France
}

\begin{abstract}
The excitation of pulsation modes in $\beta$ Cephei and Slowly Pulsating B stars is known to be very sensitive to opacity changes in the stellar interior where $T \sim 2 \times 10^{5} \mathrm{~K}$. In this region differences in opacity up to $\sim 50 \%$ can be induced by the choice between OPAL and OP opacity tables, and between two different metal mixtures (Grevesse \& Noels 1993 and Asplund et al. 2005). We have extended the non-adiabatic computations presented in Miglio et al. (2007) towards models of higher mass and pulsation modes of degree $\ell=3$, and we present here the instability domains in the HR- and $\log P-\log T_{\text {eff }}$ diagrams resulting from different choices of opacity tables, and for three different metallicities.
\end{abstract}

\section{Introduction}

The detection of B-type pulsators in low metallicity environments (see e.g. Kołaczkowski et al. 2006 and references therein), and the large number of pulsation modes detected in B stars, are now revealing new discrepancies between theory and observations that challenge standard stellar models. For instance, the two $\beta$ Cep stars 12 Lacertae and $\nu$ Eridani present low order $\mathbf{p}$-modes with frequencies higher than those predicted by pulsation models, as well as highorder g-modes (SPB type oscillation) (Jerzykiewicz et al., 2005; Handler et al., 2006, and references therein).

The interpretation of observations in the framework of standard stellar models must take into consideration the uncertainties in the basic input physics. In fact, pulsations modes in SPBs and $\beta$ Cep stars are excited by the $\kappa$-mechanism (see e.g. Dziembowski et al., 1993) due to the Fe-group opacity bump at $T \sim 2 \times 10^{5} \mathrm{~K}$, and in the last three years there have been two important 
updates of the basic physics that can affect the study of B-type pulsators: $i$ ) the revised solar metal mixture (Asplund et al., 2005) that implies a 25\% larger Fe mass fraction for a given metallicity $Z$; and $i$ i) the new Fe data included in OP opacity computations that lead to an opacity in the Z-bump increased by $18 \%$ with respect to the previous values (Badnell et al., 2005).

Miglio et al. (2007) (hereafter Paper I) and Pamyatnykh \& Ziomek (2007) showed that the combination these updates have a remarkable effect on the instability domains of SPB and $\beta$ Cephei pulsators compared to the results obtained using OPAL opacities (Iglesias \& Rogers, 1996) and Grevesse \& Noels (1993) metal mixture.

In Paper I we analyzed the role of chemical composition and opacity computations on the instability strip of B-type pulsators and on the frequency domain of expected excited modes. In the present paper we have extended the computations presented in Paper I by considering stellar masses up to $18 \mathrm{M}_{\odot}$ instead of $12 \mathrm{M}_{\odot}$, and by carrying out the non-adiabatic analysis also for $\ell=3$ modes. Only throughout comparisons with observations we will be able to assess if, and to which extent, the current uncertainties on opacity calculations and on the assumed metal mixture are able to explain the discrepancies between recent observations and standard stellar models. For this purpose we present in the following sections the instability strips in the HR $\left(\log L-\log T_{\text {eff }}\right)$ and in the period-effective temperature $\left(\log P-\log T_{\text {eff }}\right)$ diagrams resulting from the non-adiabatic calculations presented in Paper I and extended as mentioned above.

\section{Stellar models and opacities}

We computed stellar models with the code CLES (Code Liégeois d'Evolution Stellaire, Scuflaire et al. 2007). The main physical inputs are: OPAL2001 equation of state (Rogers \& Nayfonov, 2002) and Caughlan \& Fowler (1988) nuclear reaction rates with Formicola et al. (2004) for the ${ }^{14} \mathrm{~N}(\mathrm{p}, \gamma)^{15} \mathrm{O}$ crosssection. Convective transport is treated by using the classical Mixing Length Theory of convection (Böhm-Vitense, 1958), and a convective overshooting parameter of 0.2 pressure scale height was assumed in all the models. For the chemical composition we have considered: Grevesse \& Noels (1993) (GN93) and Asplund et al. (2005) corrected with the Ne abundance determined by Cunha et al. (2006) (AGS05+Ne). We have computed models with: i) OPAL opacity tables with GN93 and ii) AGS05+Ne chemical composition, then models with iii) OP opacity tables assuming GN93 and iv) AGS05+Ne mixtures. All the opacity tables are completed at $\log T<4.1$ with the corresponding GN93 and AGS05 low temperature tables by Ferguson et al. (2005).

The masses considered span from 2.5 to $18 \mathrm{M}_{\odot}$, and the chemical com- 
positions considered are: $X=0.70$ for the hydrogen mass fraction, and three different metal mass fractions: $Z=0.02,0.01$ and 0.005 . For all the models the evolution was followed from the Pre-Main Sequence.

We recall that the differences between opacities computed with OPAL, OP and with the metal mixtures considered can reach nearly $50 \%$ in the region where the driving of pulsations occurs $\left(T \sim 2 \times 10^{5} \mathrm{~K}\right)$ for a typical $\beta$ Cep star (see Paper I for a detailed comparison). Though not included in the calculations presented here, it is worth recalling that the effect of considering the Asplund et al. (2005) metal mixture without the higher Neon abundance proposed by Cunha et al. (2006) is to further increase the Fe relative mass fraction by $\sim 5 \%$. In a $10 \mathrm{M}_{\odot}$ model (and for a given value of $Z$ ) this induces a further increase of the opacity at $T \sim 2 \times 10^{5} \mathrm{~K}$ up to $7 \%$, that only slightly modifies the instability strips presented here.

\section{Results: Updated SPBs and $\beta$-Cep instability domains}

We carry out a pulsational stability analysis of main-sequence models from our grid using the non-adiabatic code MAD (Dupret et al., 2003). As mentioned above, in these computations we fixed the overshooting parameter $\alpha_{\text {ov }}$ at 0.2 and the initial hydrogen mass fraction $X$ at 0.70 . For discussion about the effect of assuming different $\alpha_{\text {ov }}$ or $X$ on the stability domain, as well as for the stability study in post-MS models, we refer to the work by Pamyatnykh (1999). We checked the stability of radial modes and of non-radial $\mathrm{p}$ - and g-modes of degree $1 \leq \ell \leq 3$.

The location of the instability strip in the HR diagram and the frequency of the excited modes are determined by the properties of the metal opacity bump. The effects of the choice of the metal mixture (GN93 or AGS05+Ne) and of the opacity computations (OPAL or OP) on the HR location of instability domains are shown in figures 1,2 , and 3 for models with metallicity $Z=0.02,0.01$, and 0.005 , respectively. The combined effects on the excited modes of OP opacity and AGS05+Ne metal mixture, compared with the standard OPAL with GN93, are also shown by means of the Period- $T_{\text {eff }}$ diagram in Fig. 4.

The results presented in these figures can be summarized as follows:

1. Since the region where $\kappa_{\mathrm{T}}=\left(\partial \log \kappa_{\mathrm{R}} / \partial \log T\right)_{\rho}$ increases outwards is found deeper in the star, with respect to the models computed with OPAL tables, the blue borders of the instability strips are hotter with OP models compared to OPAL ones.

2. The $T_{\text {eff }}$ domain for which we find SPB pulsators using OP opacities is $\sim 3000 \mathrm{~K}$ larger than for OPAL models. As a consequence, the number of expected hybrid $\beta$ Cep-SPB objects is also larger for OP models. 
3. The impact of the different OP-OPAL opacities is more important for low metallicity. As shown in Fig. 2, while OPAL-GN93 models with $\mathrm{Z}=0.01$ are hardly able to produce a narrow instability strip at the end of MS, with excited modes only for $\ell>1$, the OP models present $\ell=0-3$ excited modes already for an evolutionary state corresponding to $X_{c} \simeq 0.3$.

4. The Fe-mass fraction enhancement in the AGS05+Ne mixture, compared with GN93, has the main effect of extending towards higher overtones the range of excited frequencies.

5. Furthermore, while the different profile of $\kappa$ in OP and OPAL computations modifies the blue border of the instability strip, a larger Fe-mass fraction in the metal mixture provides a slightly wider instability bands, and this effect increases as the metallicity decreases. Thus, the number of $\beta$ Cep pulsators expected with $\mathrm{AGS05}+\mathrm{Ne}$ is more than three times larger than with GN93.

6. Computations for the lowest metallicity considered $(Z=0.005)$, show that none of the different OP/OPAL and GN93/AGS05+Ne evolutionary tracks for masses up to $18 M_{\odot}$ predicts $\beta$ Cep pulsators, whereas we find SPBtype modes excited when considering OP with AGS05+Ne.

The instability strips presented in this work will be made available to the community via the HELAS (European Helio- and Asteroseismology Network) website $^{1}$ and are also available upon request to the authors.

Acknowledgments. The authors are thankful to R. Scuflaire for his kind help with CLES. A.M. and J.M. acknowledge financial support from the ProdexESA Contract Prodex 8 COROT (C90199).

\section{References}

Asplund, M., Grevesse, N., Sauval, A. J., et al. 2005, A\&A 431, 693

Böhm-Vitense, E. 1958, ZA 46, 108

Badnell, N. R., Bautista, M. A., Butler, K., et al. 2005, MNRAS 360, 458

Caughlan, G. R., \& Fowler, W. A. 1988, Atomic Data and Nuclear Data Tables 40, 283

Cunha, K., Hubeny, I., \& Lanz, T. 2006, ApJ 647, L143

Dupret, M.-A., De Ridder, J., De Cat, P., et al. 2003, A\&A 398, 677

Dziembowski, W. A., Moskalik, P., \& Pamyatnykh, A. A. 1993, MNRAS 265, 588

\footnotetext{
${ }^{1}$ http://http://www.helas-eu.org
} 
Ferguson, J. W., Alexander, D. R., Allard, F., et al. 2005, ApJ 623, 585

Formicola, A., Imbriani, G., Costantini, H., et al. 2004, Physics Letters B 591, 61

Grevesse, N., \& Noels, A. 1993, in La formation des éléments chimiques, AVCP, ed. R. D. Hauck B., Paltani S., 205-257

Handler, G., Jerzykiewicz, M., Rodríguez, E., et al. 2006, MNRAS 365, 327

Iglesias, C. A., \& Rogers, F. J. 1996, ApJ 464, 943

Jerzykiewicz, M., Handler, G., Shobbrook, R. R., et al. 2005, MNRAS 360, 619

Kołaczkowski, Z., Pigulski, A., Soszyński, I., et al. 2006, Memorie della Societa Astronomica Italiana 77, 336

Miglio, A., Montalbán, J., \& Dupret, M.-A. 2007, MNRAS 375, L21

Pamyatnykh, A. A. 1999, Acta Astronomica 49, 119

Pamyatnykh, A. A. \& Ziomek, W. 2007, CoAst 150, 207

Rogers, F. J. \& Nayfonov, A. 2002, ApJ 576, 1064

Scuflaire, R., Théado, S., Montalbán, J., et al. 2007, Ap\&SS, in press 


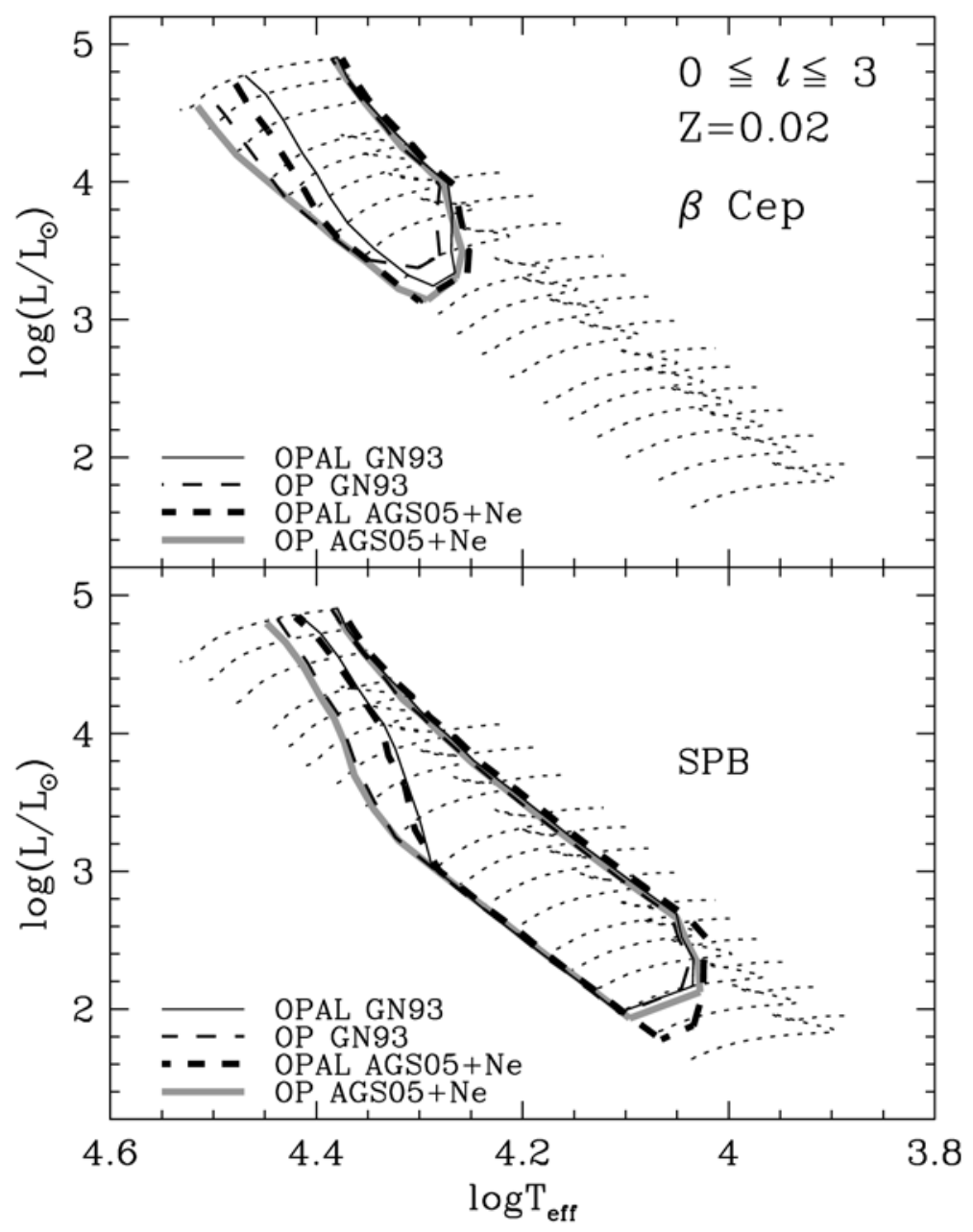

Figure 1: Instability strips of $\beta$ Cep- and SPB-type pulsations in the HR diagram for $\mathrm{Z}=0.02$. Evolutionary tracks are represented by dotted lines. 


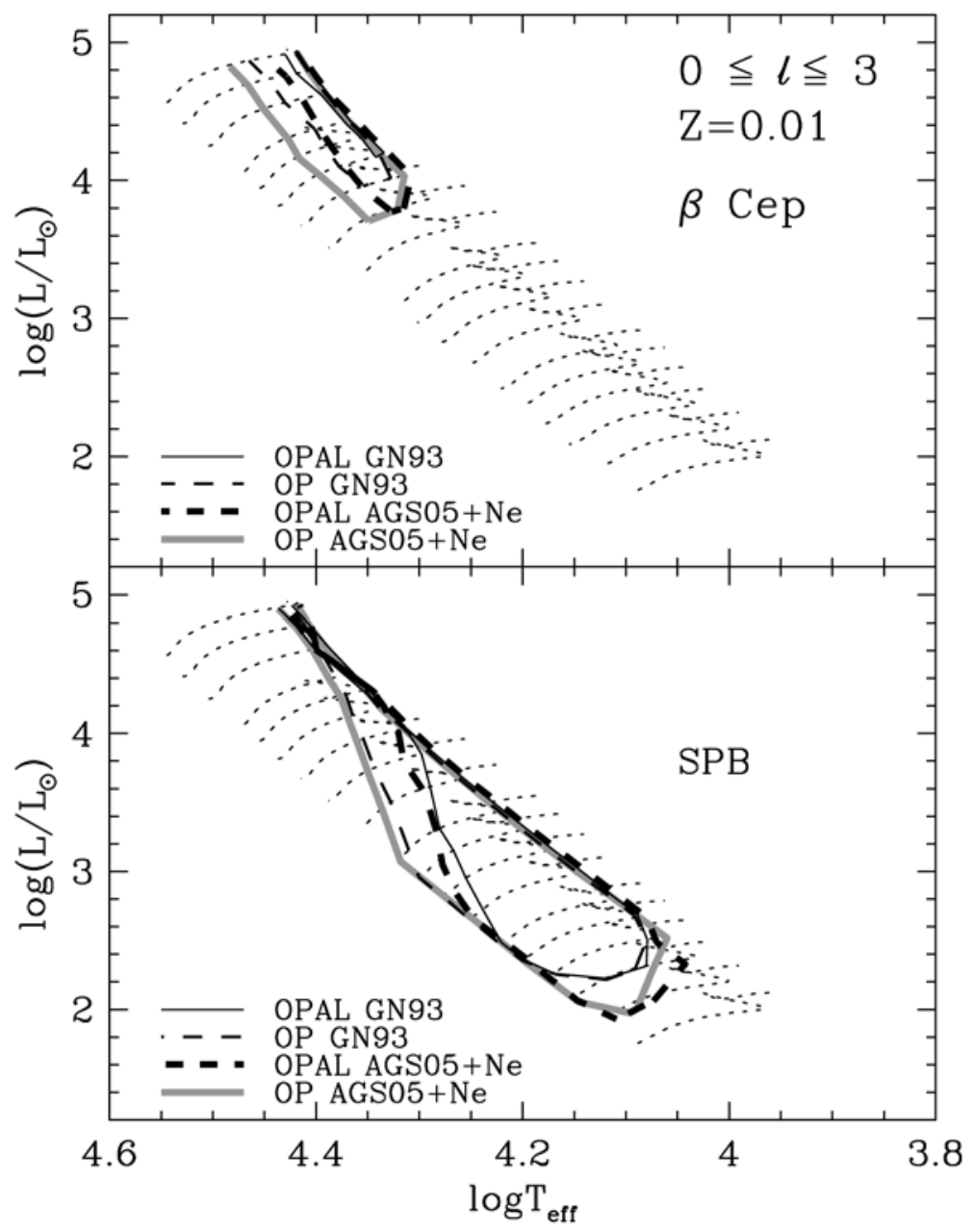

Figure 2: Same as Fig. 1 but for $Z=0.01$ 


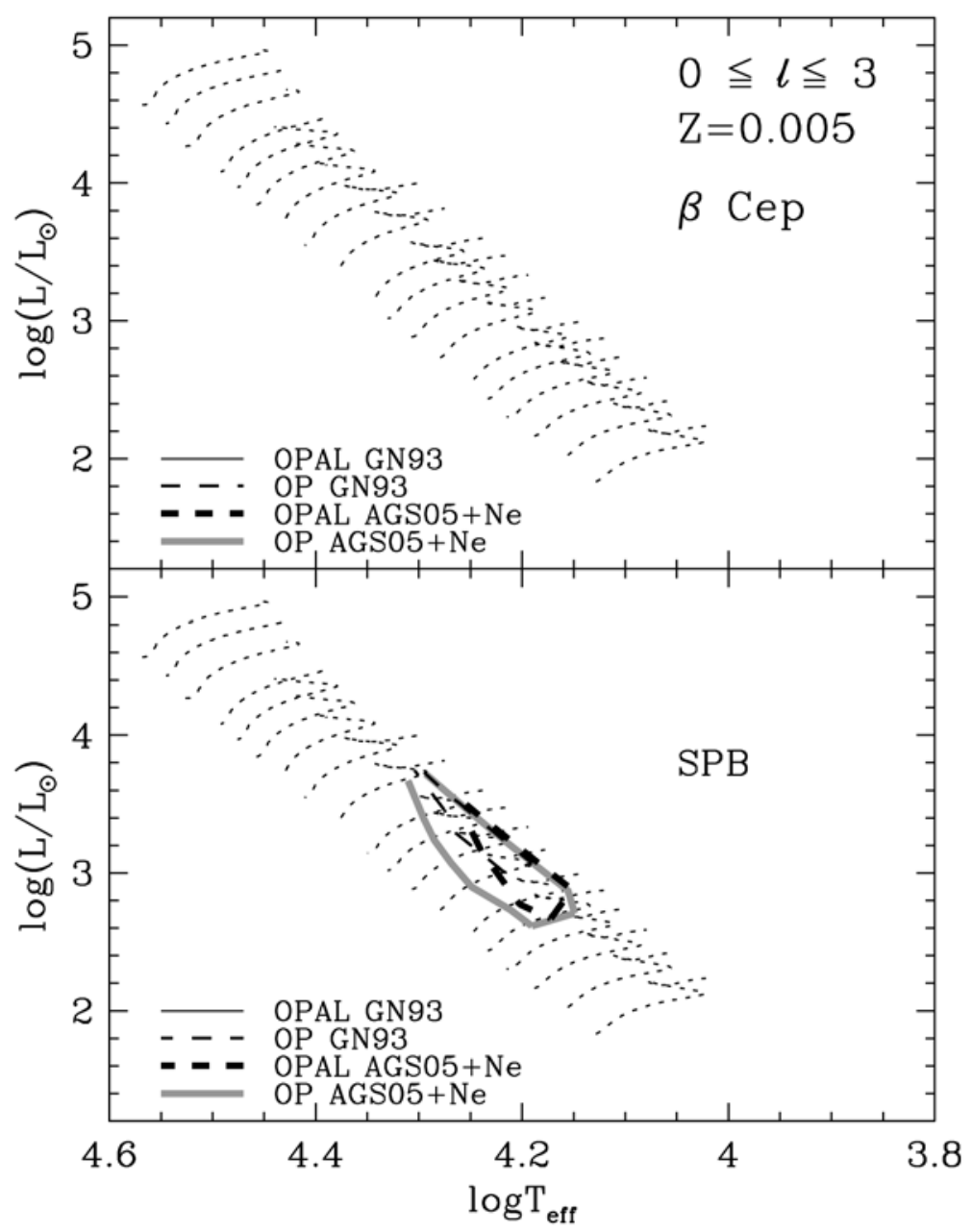

Figure 3: Same as Fig. 1 but for $Z=0.005$ 


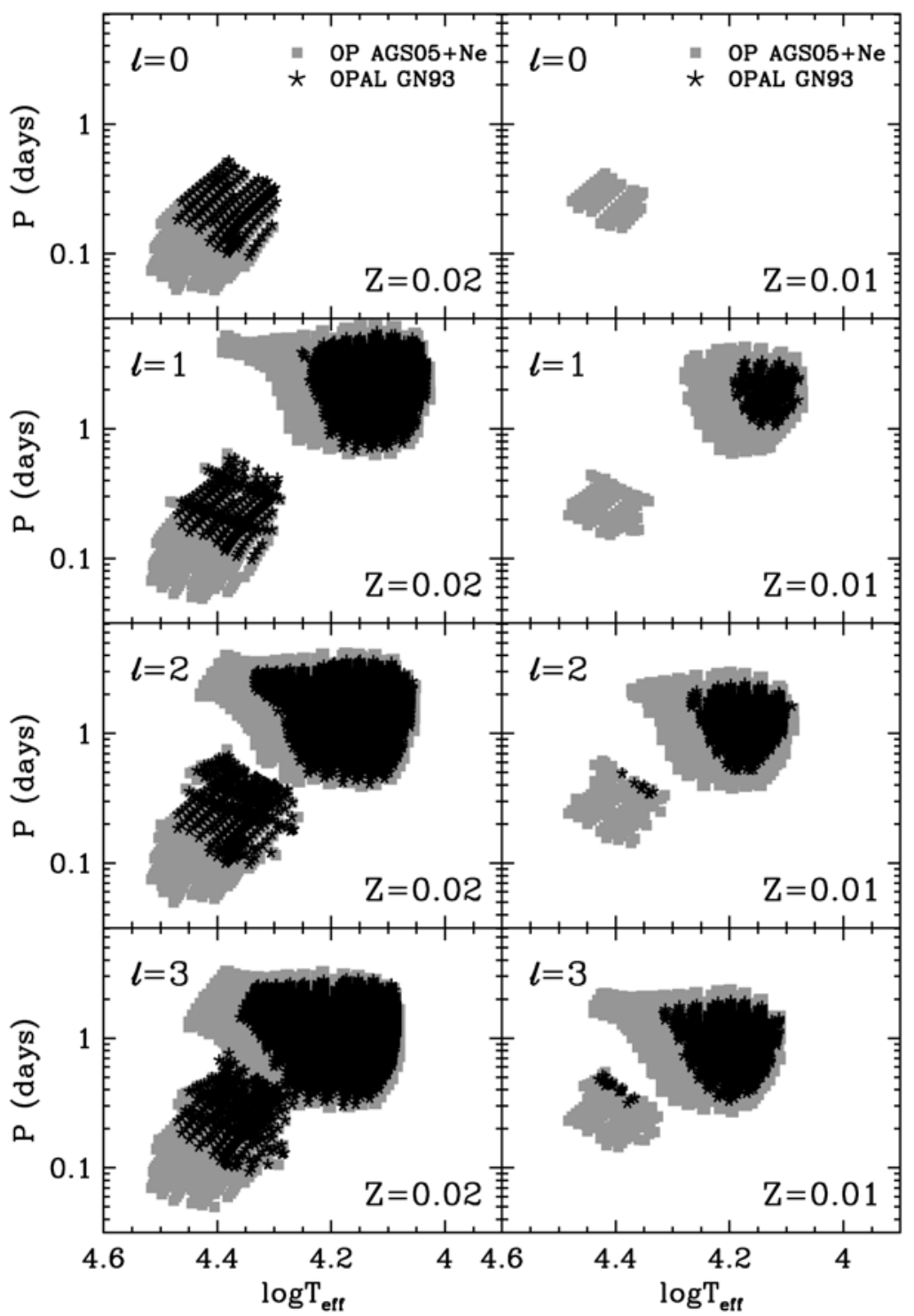

Figure 4: Instability strips represented in a $\log T_{\text {eff }}-\log P$ diagram for $\mathrm{Z}=0.02,0.01$ and different degree $\ell$. In each panel, the two regions of unstable modes represent $\beta$ Cep- and SPB-type pulsations. 